Огородников В. А.

\title{
ПОСТРОЕНИЕ ДИАГРАММ ПЛАСТИЧНОСТИ С УЧЕТОМ МЕХАНИКИ ЛОКАЛИЗАЦИИ ДЕФОРМАЦИЙ ПРИ ОДНООСНОМ РАСТЯЖЕНИИ
}

Диаграммы пластичности, используемые в феноменологических критериях разрушения в качестве характеристики материала, получают путем испытания материалов на различные виды - растяжение, сжатие, кручение или комбинация этих видов испытаний. при этом показатели напряженного состояния - безразмерные величины обычно равные отношению инвариантов тензора или девиатора напряжений должны оставаться постоянными в течение всего времени деформирования, вплоть до разрушения. Такими показателями, получившими широкое распространение в теории деформируемости, являются показатели

$$
\begin{gathered}
\eta=\frac{I_{1}\left(T_{\sigma}\right)}{\sqrt{3 I_{2}\left(D_{\sigma}\right)}}=\frac{\sigma_{1}+\sigma_{2}+\sigma_{3}}{\sigma_{u}}, \\
\chi=\frac{\sqrt[3]{I_{3}\left(T_{\sigma}\right)}}{\sqrt{3 I_{2}\left(D_{\sigma}\right)}}=\frac{\sqrt[3]{\sigma_{1} \cdot \sigma_{2} \cdot \sigma_{3}}}{\sigma_{u}}, \\
\mu_{\sigma}=2 \frac{\sigma_{2}-\sigma_{3}}{\sigma_{1}-\sigma_{3}},
\end{gathered}
$$

где $I_{1}\left(T_{\sigma}\right), I_{3}\left(T_{\sigma}\right), I_{2}\left(D_{\sigma}\right)$ - соответственно первый, третий инварианты тензора напряжений, второй инвариант девиатора напряжений, $\sigma_{1}, \sigma_{2}, \sigma_{3}$ - главные напряжения, $\sigma_{u}$ - интенсивность напряжений. Экспериментально построенные кривые $e_{p}\left(\eta, \chi, \mu_{\sigma} \ldots\right)$

$$
e_{p}=\int d \varepsilon_{u}
$$

аппроксимируют, и полученные функции называют диаграммой пластичности [1-5]. При проведении экспериментов необходимо соблюдать условия $\eta=$ const, $\chi=$ const, $\mu_{\sigma}=$ const. Однако указанное условие постоянства показателя напряженного состояния в процессе испытания часто не выполняется. Путь деформирования частиц материала $\eta\left(e_{u}\right)$, например, при растяжении может изменяться от $\eta=1$ (одноосное растяжение) до $\eta>2$ (двухосное растяжение). Как показано в работах [4-5] предельная деформация оказывается, зависящей от истории деформирования и в некоторых случаях превышает величину $e_{p}(\eta=0)$ (кручение), что противоречит физическим представлениям.

Кроме того, как показано в работе [5] при растяжении образцов из материалов, разрушению которых предшествует локализация деформаций в виде "шейки" на величину $e_{p}(\eta=1)$ оказывает влияние объемность схемы напряженного состояния. В качестве показателя напряженного состояния в работе [5] предложен показатель (2), учитывающий влияние третьего инварианта тензора напряжений на пластичность в условиях локализации деформаций $e_{p}(\eta=1)$. В этой же работе показано, что $I_{3}\left(T_{\sigma}\right)$ оказывает влияние на пластичность. В области изменения показателя $1 \geq \eta \geq-2$ на диаграмму пластичности оказывает влияние третий инвариант тензора напряжений в области $1>\eta>0$ третий инвариант повышает пластичность (рис. 1) (по сравнению с диаграммой пластичности, построенной в условиях $I_{3}\left(T_{\sigma}\right)=0$ ). 


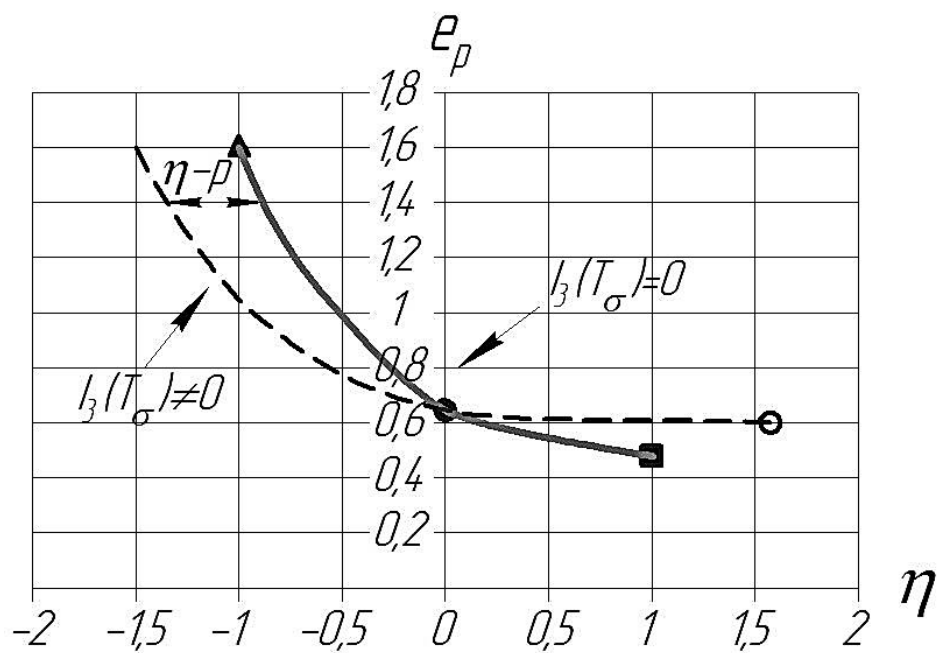

Рис. 1. Диаграмма пластичности стали 20:

- - растяжение; $\boldsymbol{\Delta}$ - сжатие; • - кручение; ○- $\eta=1,55$---- $I_{3}\left(T_{\sigma}\right) \neq 0,-I_{3}\left(T_{\sigma}\right)=0$,

Как показано в работе [6] при постоянном показателе напряженного состояния градиент деформаций повышает пластичность. В указанной работе приведены результаты экспериментов, проведенных на образцах из быстрорежущих сталей Р12 и Р18 квадратного и прямоугольного поперечного сечений. Различный градиент деформаций достигается за счет различных размеров поперечного сечения. Образцы деформировали в условиях чистого изгиба вплоть до разрушения. Деформации определяли экспериментально методом делительных сеток, нанесенных с помощью твердомера "Виккерс" базой 1 мм на боковую поверхность.

Степень деформации при чистом изгибе:

$$
e_{u}=\frac{\sqrt{2}}{3} \sqrt{\left(\varepsilon_{r}-\varepsilon_{\theta}\right)^{2}+\left(\varepsilon_{\theta}-\varepsilon_{Z}\right)^{2}+\left(\varepsilon_{Z}-\varepsilon_{r}\right)^{2}} .
$$

Тензор деформаций, характеризующий градиент деформаций:

$$
T_{3}=\sqrt{\left(\frac{\partial \varepsilon_{r}}{\partial r}\right)^{2}+\left(\frac{\partial \varepsilon_{\theta}}{\partial r}\right)^{2}+\left(\frac{\partial \varepsilon_{z}}{\partial r}\right)^{2}+2\left(\frac{\varepsilon_{r}}{r_{0}}-\frac{\varepsilon_{\theta}}{r_{0}}\right)^{2}} .
$$

Производные определяли как тангенс угла наклона касательных к соответствующим зависимостям в точках примыкающих к месту разрушения. Радиусы кривизны определяли для растянутой поверхности в области примыкающей к линии разрыва.

Показатель напряженного состояния (1) равный отношению первого инварианта тензора напряжений в интенсивности напряжений рассчитывали по формуле:

$$
\eta=2 \frac{\varepsilon_{r}}{e_{u}}
$$

Значение $\eta$ практически не отличалось от единицы.

На основе обработки полученных экспериментальных данных в работе [6] исходя из теории диффузии дислокаций и предположения о том, что при $\eta=$ const интенсивность деформаций пропорциональна плотности дислокаций, предложено следующее уравнение:

$$
e_{p}=b \exp (\beta t)+\frac{4}{3} \frac{\frac{\partial e_{u}}{\partial x} \int_{x=l} \sqrt{D t}}{\sqrt{\pi}} .
$$


Зависимость (8) проверена экспериментами: $\beta t=2,0816, D t=16,81 \mathrm{mм}^{2}, b=0,0095$. Экспериментальные данные аппроксимировали выражением:

$$
e_{p}=0,5\left(\operatorname{grad} e_{u}\right)^{0,31}
$$

или

$$
\operatorname{grad} e_{u}=\exp \left(\ln \frac{e_{u}}{0,5} / 0,31\right)
$$

Полученные результаты дают возможность учесть влияние градиента деформаций при построении диаграммы пластичности.

В работе [7] исследована предельная деформация на контуре центрального кругового отверстия в растягиваемых пластинах шириной 55 мм и толщиной 2 мм из стали Ст.3. Пластины растягивали ступенчато возрастающей нагрузкой. На пластинах нанесена делительная сетка базой 0,1 мм. Измеряли удельные деформации $e_{u}$.

Полученные результаты зависимости предельной интенсивности деформаций от градиента деформаций аппроксимировали соотношением:

$$
e_{p}=B\left(\operatorname{grad} \frac{1}{M M}\right)^{m},
$$

или

$$
\operatorname{grad} e_{u} \frac{1}{M \mu}=\exp \left(\ln \frac{e_{p}}{B} / m\right)
$$

где $B=0,85, m=0,261$.

Наибольшая неоднородность напряженно-деформированного состояния оказывается также при таком виде испытаний как кручение $(\eta=0)$.

При постоянстве показателя напряженного состояния $(\eta=0)$ реализовали испытанием на кручение образцов диаметром $d=20,15,10,7,5,5$ мм.

Вдоль образующих цилиндрических образцов с помощью штангенциркуля проводили продольную риску. После разрушения на инструментальном микроскопе измеряли углы наклона винтовых линий вблизи места разрушения. Степень деформации определяли по формуле:

$$
e_{p}=\frac{\operatorname{tg} \alpha}{\sqrt{3}}
$$

Измеряли также диаметр образца и длину рабочей части до и после разрушения. В рассматриваемом случае эти параметры практически не изменялись. Следовательно, кручение было стесненным, т. е. возникали осевые сжимающие напряжения, однако как показано в работе [7] они на два порядка меньше касательных и удлинение образцов практически отсутствовало. Следовательно, гипотеза плоских сечений выполняется $\left(\varepsilon_{\mathrm{z}}(\mathrm{z})=\mathrm{const} \cong 0, \sigma_{\mathrm{z}} \cong 0\right)$.

Квадратичный инвариант тензора деформаций $T_{3}$ при кручении равен:

$$
T_{3}=\left[\left(\frac{\varepsilon_{z} \theta}{r}\right)^{2}+\left(\frac{\partial \varepsilon_{z} \theta}{\partial r}\right)^{2}\right]^{\frac{1}{2}} .
$$


При кручении $\operatorname{grad} \varepsilon_{u}=\frac{d e_{u}}{d r} \cdot \frac{1}{e_{u \max }}=\frac{d \gamma}{d r} \cdot \frac{1}{\gamma_{\max }}$, т. е. при радиусе $r=4$ мм grad $\varepsilon_{u}=\frac{1}{r}=0,25 \frac{1}{\text { мм }}$ влияние градиента деформаций несущественно.

Среди многочисленных аппроксимаций диаграмм пластичности $[1-5,7,8]$ остановимся на тех, у которых коэффициенты аппроксимации имеют физический смысл. Так в работах [2-5] приведена аппроксимация вида:

$$
e_{p}(\eta)=e_{p}(\eta=0) \exp \left(-\lambda_{i} \eta\right)
$$

Здесь коэффициент аппроксимации $\lambda_{i}$ равен:

$$
\lambda_{i}=\ln \frac{e_{p}(\eta=0)}{e_{p}(\eta=1)} .
$$

Его использование в уравнении (15) необходимо в пределах изменения показателя в пределах $1 \geq \eta>0$. Для интервала изменений показателя $0 \geq \eta \geq-1$ коэффициент $\lambda_{2}$ равен:

$$
\lambda_{2}=\ln \frac{e_{p}(\eta=-1)}{e_{p}(\eta=0)} .
$$

На рис. 2 показана диаграмма пластичности быстрорежущей стали Р18, аппроксимированная с помощью формулы (15), где $\lambda_{1}=1,31, \lambda_{2}=1,26$. Данная марка стали была выбрана в связи с тем, что при растяжении не образуется шейка.

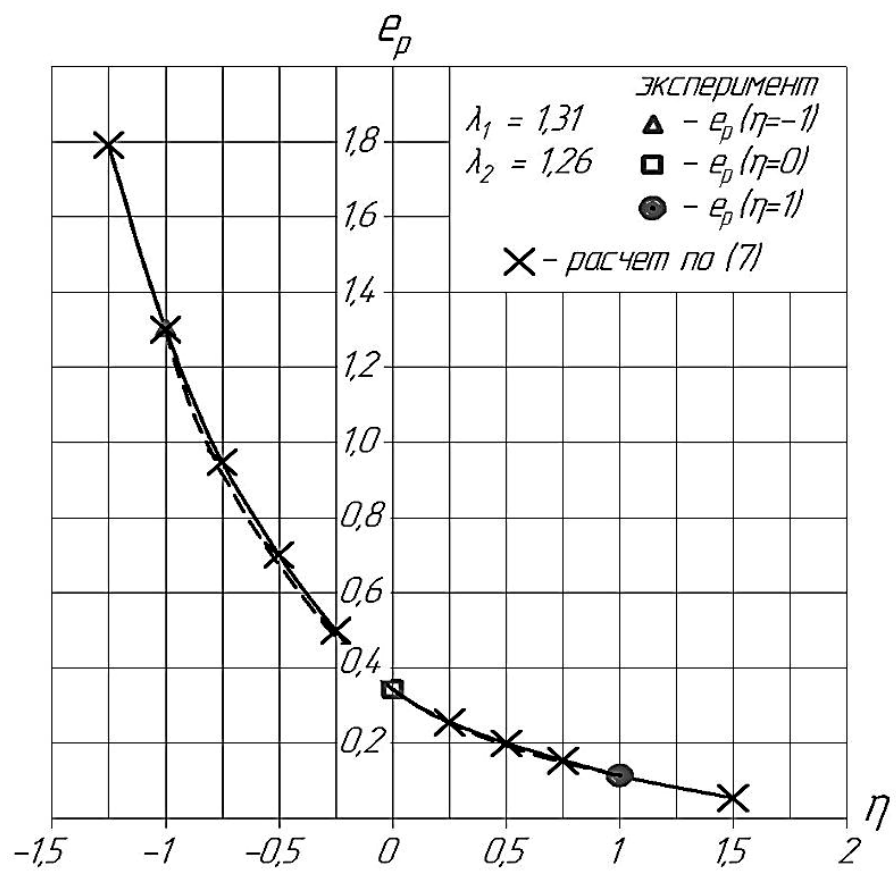

Рис. 2. Диаграмма пластичности быстрорежущей стали Р18

В полулогарифмических координатах $\lambda_{i}$ - тангенсы угла наклона прямых, построенных в координатах $e_{p}(\eta)$. Коэффициент $\lambda_{i}(i=1,2)$ - это по существу коэффициент "чувствительности" пластичности к изменению схемы напряженного состояния. Чем больше величина этих коэффициентов, тем интенсивнее происходит рост пластичности с возрастанием гидростатического давления. 
Подобное представление о физической сущности коэффициентов $\lambda$ позднее опубликовано в монографии В. А. Скуднова [8], в которой с точки зрения физики металлов показана целесообразность введения коэффициентов "чувствительности" пластичности к изменению показателя $\eta$. Так в указанной работе перегибы на диаграммах пластичности, построенных в полулогарифмических координатах, связаны с показателем релаксационной способности (пластичности) поликристаллов $m$ :

$$
m=\frac{d \ln \sigma}{d \ln \dot{\varepsilon}},
$$

а величины $e_{p}, \delta, \psi$ есть следствие величины $m$ (но не только ее одной).

Таким образом, в настоящей работе рассмотрены особенности, возникающие при построении диаграмм пластичности в условиях таких испытания, как одноосное растяжение, а также кручение. На примере стали Ст. 3, испытанной в условиях растяжения, рассмотрим предложенный нами алгоритм построения диаграмм пластичности в интервале изменения показателя $\eta$ от $\eta=0$, до $\eta=2$ [9].

Три стандартных цилиндрических образца из стали Ст. 3 (данная сталь выбрана в связи с тем, что разрушению этой стали предшествует локализация в виде шейки) растягивали до различных степеней деформаций $\left(\delta=\frac{l_{i}-l_{0}}{l_{0}} \cdot 100 \%, \delta_{1}=2,56 \%, \delta_{2}=15,92 \%, \delta_{3}=20,92 \%\right)$. После деформации (3-й образец был разрушен) измеряли геометрические параметры (см. табл. 1).

Таблица 1

Геометрические параметры и некоторые характеристики образцов стали Ст. 3

\begin{tabular}{|c|c|c|c|c|c|c|c|c|c|c|c|c|}
\hline $\begin{array}{c}\mathrm{N} \\
\text { обр. }\end{array}$ & $l$, мм & $\begin{array}{c}h, \\
\text { мм }\end{array}$ & $\begin{array}{c}d_{u}, \\
\text { мм }\end{array}$ & $\begin{array}{c}d_{0}, \\
\text { мм }\end{array}$ & $\begin{array}{c}d_{y c m .}, \\
\text { мм }\end{array}$ & $\begin{array}{c}l_{0}, \\
\text { мм }\end{array}$ & $\delta, \%$ & $\begin{array}{c}R, \\
\text { мM }\end{array}$ & $r$, мм & $e_{p}$ & $d_{\text {nред }}$ & $e_{p}$ \\
\hline 1 & 12 & 4,675 & 5,1 & 9,61 & 8,00 & 9,926 & 20,9 & 11,58 & 1,3 & 1,27 & 5,9 & 0,975 \\
\hline 2 & 14 & 1,1 & 6,1 & 9,61 & 8,05 & 9,926 & & 22,82 & 1,19 & 0,91 & 6,35 & 0,829 \\
\hline 3 & 16 & 1 & 7,7 & 9,61 & 8,45 & 9,926 & & 34 & 1,165 & 0,443 & 7,27 & 0,558 \\
\hline
\end{tabular}

Следуя работе [9] радиус шейки определяли по формуле:

$$
R=\frac{l^{2}+4 h^{2}}{8 h}
$$

Показатель напряженного состояния по Бриджмену:

$$
\eta=1+3 \ln \left(1+\frac{d_{i}}{4} \cdot \frac{1}{R}\right)
$$

В работе [10] показан снимок шейки алюминиевого образца непосредственно перед разрушением. При этом в центре сечения наблюдается макротрещина, которая не дошла до краев контура сечения. Поэтому расчет предельной деформации по формуле

$$
e_{p}=2 \ln \frac{d_{0}}{d_{u}}
$$

существенно завышает пластичность.

В работе [9] предлагается расчет предельной деформации $e_{p}$ проводить по формуле: 


$$
e_{p}^{*}=2 \cdot \ln \frac{d_{0} \cdot 2,22}{d_{u}+d_{y c m}}
$$

где $d_{y c m}$ - диаметр сечения цилиндрического образца на удалении от минимального радиуса шейки.

Расчет по (22) занижает пластичность, что соответствует физическим представлениям о падении пластичности с увеличением показателя $\eta$.

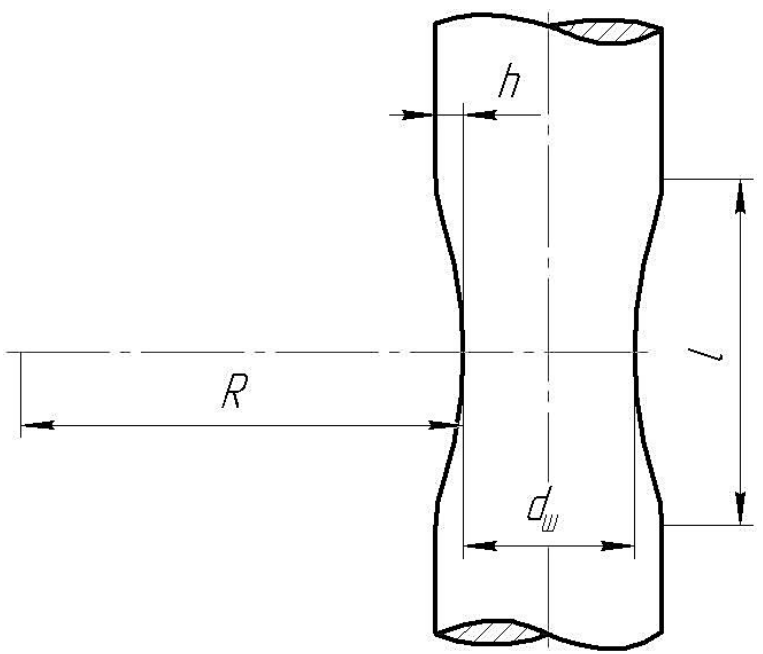

Рис. 3. Схема для определения показателя напряженного состояния $\eta$ и радиуса контура шейки $R$

Дадим обоснование возможности такого расчета на основе изложенного выше подхода о влиянии трех факторов на пластичность - градиента деформаций, истории деформирования и третьего инварианта тензора напряжений.

В рассматриваемом примере растяжения цилиндрических образцов из стали Ст. 3 в области локализации деформации (в шейке) градиент деформаций завышает пластичность на величину $\Delta e_{u} \approx 0,11$, история деформирования $\omega=\frac{e_{p}(\eta)}{e_{p}(\eta=\text { const })}=1,12 \ldots 1,14$, третий инвариант $I_{3}\left(T_{\sigma}\right)$ завышает пластичность на 10-12\%. Расчет фактической предельной деформации дает результат:

$$
e_{p}^{*}=e_{p}[1-(0,11+0,13+0,11)]=1,27[1-0,35]=0,8255 \text {. }
$$

Расчет по формуле (22) дает удовлетворительную сходимость с расчетом по (23) $e_{p}^{*}=0,975$.

\section{ВЫВОДЫ}

Разработана методика расчета предельных деформаций при построении диаграмм пластичности в условиях локализации деформации при одноосном растяжении. «Аномальное» повышение пластичности при возрастании показателя напряженного состояния объясняется влиянием трех факторов: градиента деформаций, истории деформирования и третьего инварианта тензора напряжений.

На примере построения диаграммы пластичности стали Ст. 3 показано количественное влияние указанных трех факторов на величину предельной деформации в шейке растянутого до разрушения образца. Суммарное значение завышения предельной деформации составляет $35 \%$. 


\section{СПИСОК ИСПОЛЬЗОВАННОЙ ЛИТЕРАТУРЫ}

1. Огородников В. А., Деревенько И. А., Побережный М. И. Карты материалов в процессах обработки материалов давлением. Вісник національного технічного університету України "КПI". Серія "Машинобудування". 2011. № 62.С. 88-91.

2. Огородников В. А., Алиева Л. И., Кожушаный В. М., Деревенько И. А. Параметры модели, формирующей карту материала в процесах обработки давлением. Обработка материалов давлением. Краматорск : ДГМА, 2011. № 1(26). С. 91-98.

3. Огородников В. А., Алиева Л. И., Деревенько И. А. Параметры напряженного состояния диаграмм пластичности. Обработка материалов давлением. Краматорск : ДГМА, 2011. № 4(29). С. 10-18.

4. Del G. D., Ogorodnikov V. A., Spiridonov L. K. Plasticity of Metal Subjected to Complex [Article plastichnost' metalla pri slozhnom nagruzhenii]. Izv. Vyssh. Uchebn. Zaved. Mashinostr. 1974. № 12. P. 22-26.

5. Огородников В. А. Деформируемость и разрушение металлов при пластическом формоизменении. Киев: УМК ВО, 1989. 152 с.

6. Огородников В. А., Сивак И. О. Зависимость пластичности металлов от градиента пластических деформаций. Изв. АН СССР. Металльл. 1978. № 6. С. 169-174.

7. Дель Г. Д. Технологическая механика. Москва: Машиностроение, 1978. 174 с.

8. Скуднов В. А. Предельные пластические деформации металлов. Москів: Металлургия, 1989. 176 с.

9. Огородніков В. А., Кириця І. Ю., Перлов В. Є. Механіка процесів холодного пластичного деформування вісесиметричних заготовок з глухим отвором: монографія. Вінниця: ВНТУ, 2015. 164 с.

10. Малинин Н. Н. Прикладная теория пластичности и ползучести. Москва: Машиностроение, 1975. 400 с.

\section{REFERENCES}

1. Ogorodnikov V.A., Derevenko I.A., Poberezhny M.I. Maps of materials in the processes of processing materials by pressure. Bulletin of the National Technical University of Ukraine "KPI". Series "Mechanical Engineering". 2011. No. 62. 88-91. (in Russian).

2. Ogorodnikov V.A., Alieva L.I., Kozhushany V.M., Derevenko I.A. Parameters of the model forming the map of the material in the pressure processing. Materials working by pressure. Kramatorsk: DSMA, 2011. No. 1 (26). S. 91-98. (in Russian).

3. Ogorodnikov V.A., Alieva L.I., Derevenko I.A. Parameters of the stress state of plasticity diagrams. Materials working by pressure. Kramatorsk: DSMA. 2011. No. 4 (29). S. 10-18. (in Russian).

4. Del G.D., Ogorodnikov V.A., Spiridonov L.K. Plasticity of Metal Subjected to Complex [Article plastichnost' metalla pri slozhnom nagruzhenii]. Izv. Vyssh. Uchebn. Zaved. Mashinostr. 1974. № 12. P. 22-26.

5. Ogorodnikov V.A. Deformability and destruction of metals in plastic form change. Kyiv: UMK VO. 1989. 152 p. (in Russian).

6. Ogorodnikov V.A., Sivak I.O. Dependence of plasticity of metals on the gradient of plastic deformations. Izv. Academy of Sciences of the USSR. Metals. 1978. No. 6. P. 169-174. (in Russian).

7. Del G.D. Technological mechanics. Moscow: Mechanical Engineering. 1978. 174 p. (in Russian).

8. Skudnov V.A. Limit plastic deformations of metals. Moscow: Metallurgy, 1989. 176 p. (in Russian).

9. Ogorodnikov V.A., Kiritsa I.Y., Perlov V.E. Mechanics of cold plastic deformation of axisymmetric blanks with a blind hole: a monograph. Vinnytsia: VNTU. 2015. 164 p. (in Ukrainian). (in Russian).

10. Malinin N.N. Applied theory of plasticity and creep. Moscow: Mechanical Engineering, 1975. 400 p.

Огородников В. А. - - д-р техн. наук, проф. ВНТУ;

Сухоруков С. I. - - канд. техн. наук, доц. ВНТУ;

Архипова Т. Ф. - канд. техн. наук, доц. ВНТУ.

ВНТУ - Винницкий национальный технический университет, г. Винница.

E-mail: va.ogorodnikow@gmail.com. 